\title{
Developing an OGC Standard-Based Platform for Integration of Radiation Monitoring Data from Fukushima Area, Japan
}

\author{
Chen-Yu Hao ${ }^{1}$, Mei-Shin Chen ${ }^{1}$, Tien-Yin Chou ${ }^{1}$, Chia-Cheng Lin $^{1}$, Kim Kyoungsook ${ }^{2}$, \\ Nguyen Xuan Linh ${ }^{1}$ \\ ${ }^{1}$ GIS Research Center, Feng Chia University, Taichung, Taiwan \\ ${ }^{2}$ Artificial Intelligence Research Center, AIST, Tokyo, Japan
}

\section{Email address:}

how@gis.tw (Chen-Yu Hao)

\section{To cite this article:}

Hao Chen-Yu, Chen Mei-Shin, Chou Tien-Yin, Lin Chia-Cheng, Kim Kyoungsook, Nguyen Xuan Linh. Developing an OGC Standard-Based Platform for Integration of Radiation Monitoring Data from Fukushima Area, Japan. Radiation Science and Technology.

Vol. 4, No. 3, 2018, pp. 12-21. doi: 10.11648/j.rst.20180403.11

Received: August 19, 2018; Accepted: September 12, 2018; Published: November 14, 2018

\begin{abstract}
Geographical location of Japan is a reason that causes this country to become one of the most disaster-prone nation in the world. The Nuclear Emergency Response Headquarters of Japan Government has established a comprehensive plan for monitoring radiation due to the Tohoku earthquake on 11 March 2011 that caused the release of radioactive materials from Fukushima Daiichi Nuclear Power Station (NPS). The main purpose of this research is to develop a prototype platform based on Open Geospatial Consortium (OGC) international standards for integrating the radiation monitoring results from Fukushima Area, Japan. Because of the monitoring data was mainly stored in XML or CSV format, this platform provided a simple and standardized environment for converting original data into uniform format with three primary information including observation time, observation locations, and observation results. After stage of data standardization, all converted data was published as services utilizing OGC standards of Catalogue Service for the Web (CSW), Sensor Observation Service (SOS), Web Map Service (WMS) and JSON-based RESTful Application Program Interface (API). Finally, this platform visualizes the monitoring results and provides a client with the functionality of time series selection. The idea of this prototype platform in releasing the radioactive materials monitoring data using international standards has matured over time and does not exclude the possibility of making it open access. The result of this study has shown that the utilization of international standard is a necessary step for data integration and data sharing.
\end{abstract}

Keywords: Interoperability, SOA, Data Portal, Radioactive Materials, Fukushima Area

\section{Introduction}

Located on the east side of Pacific Ocean, in Fukushima prefecture, Fukushima Daiichi Nuclear Power Station (NPS) a station operated by Tokyo Electric Power Company (TEPCO) is the source of emission of radioactive materials due to strong earthquake and tsunami happened on 11 March 2011 in Japan [1]. Tens of thousands of citizens have been affected by this emission and had to evacuate from their home. In that situation, The Japanese government was really concerned about the level of radioactive contamination because a large area has been subject to the contamination and
Fukushima residents also needed to know whether their health was guaranteed [2].

The Nuclear Emergency Response Headquarters, from the event of Fukushima plant, has been established a comprehensive plan for monitoring radioactive substances together with many studies on contamination tracking from scientists. In the efforts of monitoring radioactive substances, Nihei in 2016 gathered samples and used a detector to discover level of radiocesium concentration on agricultural, livestock, forest and fishery products for three years from accident of NPS in 2011. The results then were published to citizens via websites of the Ministry of Health, Labor, and Welfare along with homepage of Fukushima prefecture. Also 
relating to the field of agriculture, Monma attempted to construct a monitoring system [4], which concentrated on farmland parcel of each owner for radioactive contamination parameters. This study was conducted in Tamano district locating near Fukushima city and obtained some active results. However, one disadvantage of this study is that an important result data - a map displaying the concentration of radioactive substances detailing to each parcel of farmland for whole research area was only available to people through the way of showing at public building halls. Therefore, citizens who are interested in practical results from this research will meet difficulties in access. Some other successful studies trying to monitor the level of radioactive contamination along with its impact on many of the aspects of life can be considered, for instance, Stein (2017) had monitored the status of beef [5], Ohte et al. (2016) was concerned about movement of radiocesium in a forested catchment, Matsuda et. al. (2017) implemented air dose rate measurement of both inside and outside of nearly 200 houses in Fukushima [7], and Martin et. al. (2016) tried to employ Unmanned Aerial Vehicle (UAV) to create $3 \mathrm{D}$ map of radiation for evaluating the distribution of contaminant [8].

Indeed, there are many researches and projects in monitoring the level of radioactive contamination as well as impacts on the health of citizens has been performed from Fukushima event. Monitoring results, nevertheless, come from various sensors with many of the data formats causing the difficulties in data integration and data sharing. Regarding the development of solutions for overcoming the data integration and data sharing issue, some authors select an approach of applying international standards. Hussain et al. (2015) and Anzaldi et al.(2014) utilized Open Geospatial Consortium (OGC) standards that named as WaterML 2.0, which is a model for storing water observation data. Sensor Observation Service (SOS), it defines a web service interface for managing and querying types of sensor data along with some other standards to develop a generic framework for integrating data in water distribution system [9, 10]. The authors have proved that with the combination of common knowledge and standards from OGC, the interoperability is enabled. Song et al. (2008) shared same point of view when proposing an architecture based on OGC standards for distributing services relating to coal mine maps [11]. Instead of concentrating on data of any specific fields, some researchers presented the way that international standards of OGC can be applied for enhancing the interoperability of geospatial data $[12,13]$. Other studies have also been dedicated studies on using international standards for addressing the issue of data interoperability [14, 15, 16].

Currently, Japanese government has been managing a large amount of observation data, which has been collected mainly from diverse types of sensors. Data gathered from various monitoring sensors in Fukushima may be grouped into some common categories such as general environment (soil, water, air...), schools, farmlands, food... and generally, these observed data may be divided into two primary types consisting of mobile and fixed observations. However, there is the fact that, the number of observation data produced by sensors in Fukushima is extremely large. Taking fixed observations as an example, there are more than 4000 fixed sensors, which the data collection occurred every ten minutes around NPS location. This means the amount of data obtained will exceed 200,000 records per hour. Therefore, a solution for meeting the need of data integration and data sharing in Fukushima is a natural arising.

This paper was conducted in an attempt to develop a prototype framework with the application of various OGC international standards such as Catalogue Service for the Web (CSW), Sensor Observation Service (SOS), Web Map Service (WMS) and JSON-based RESTful Application Program Interface (API) for integrating diverse kinds and large amount of radiation monitoring data in Fukushima. The citizens can visualize and query all results via an interface that provides functionality of time series for users selecting time slot of interest. Finally, conclusions and future works is presented in the last section.

\section{Materials and Methods}

\subsection{Study Area}

The monitored data utilized in this study is from Fukushima area (Figure 1) - one of three biggest prefectures in Japan with area of 13,782 square kilometer and population of about $1,884,646$ people [19].

\subsection{Data Source}

The comprehensive monitoring plan from Japan government has led to the large amount of data from various monitoring devices. In this study, the gathered data was classified into seven main categories, namely:

(1) Group 1: General environment (soil, water, air...);

(2) Group 2: School;

(3) Group 3: Ports, airports, parks;

(4) Group 4: Natural Park, waste;

(5) Group 5: Farmland, forest, pasture;

(6) Group 6: Tap water;

(7) Group 7: Food;

For further classification, based on characteristics of data acquisition, the data was grouped into either mobile or fixed types. The mobile types are normally measured by a mobile carrier with some sensors while the fixed types tend to be acquired by sensors that are attached to a fixed specific position for long-term monitoring purpose. The characteristics of the mobile data are short time period, high density, but covering a large geographical area while the fixed data is long time periods and covering a less geographical area.

The results monitored by sensors were stored in their respective file formats. In this study, more than 30 file formats were processed with the number of mobile observed data exceeded 80 million observations while the fixed type exceeded 4 million. 

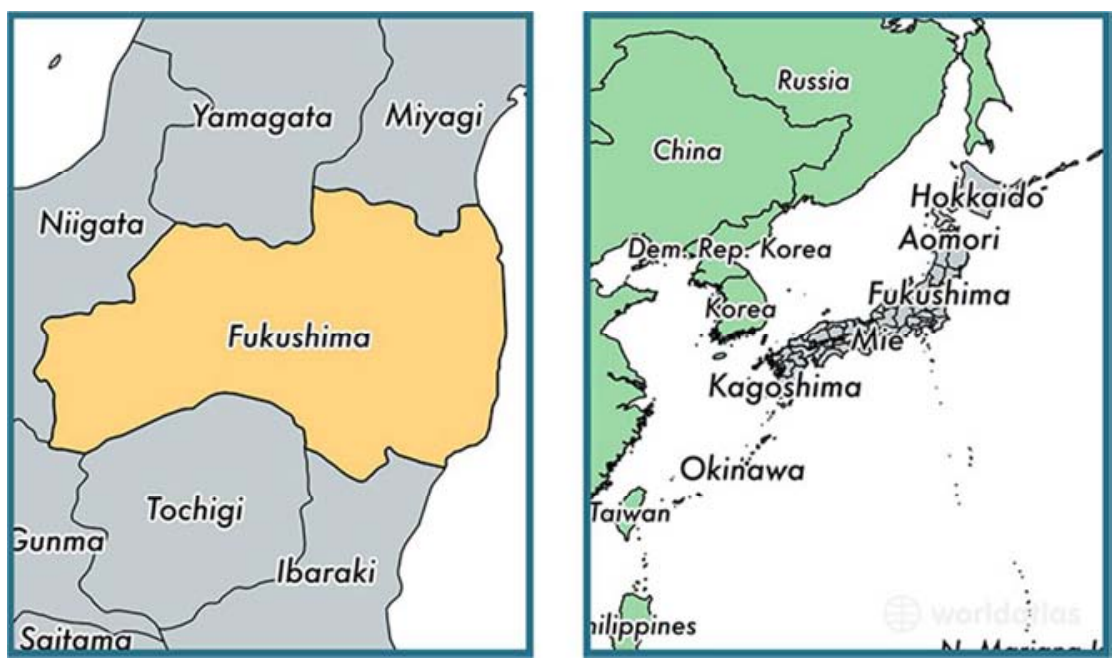

Figure 1. Location of Fukushima prefecture, Japan.

\subsection{Methodology}

In terms of geospatial data interoperability, Geospatial Web Services (GWS) is one the most used technologies providing a new means and new opportunities for accessing a large amount of geographic data and computing resources via internet environment [17]. In order to implement these tasks, standards from Open Geospatial Consortium (OGC) are popular choices from geospatial researchers [18]. OGC is composed of many government agencies, companies and universities all over the word and is a nonprofit organization established for the purpose of producing quality geospatial standards and making them open for global community. This research leveraged the standards of CWS, SOS, WMS and
JSON API from the OGC organization to construct platform.

For the purposes of storing results, publishing as OGC services and exchanging with third parties, this study has designed a portal named "Radiation Portal" for management of all tasks. There were two major phases called "Radiation Normalization" and "Radiation Publication" from the stage of data processing to data publishing as services (Figure 2). The portal provides functionalities of importing files from CSV or XML formats as data sources, stores them in a server as a normalized database and then converting into different kinds of formats such as SHP, GPX or KML for various purposes of usage. After importing data, users can select type of service for publication.
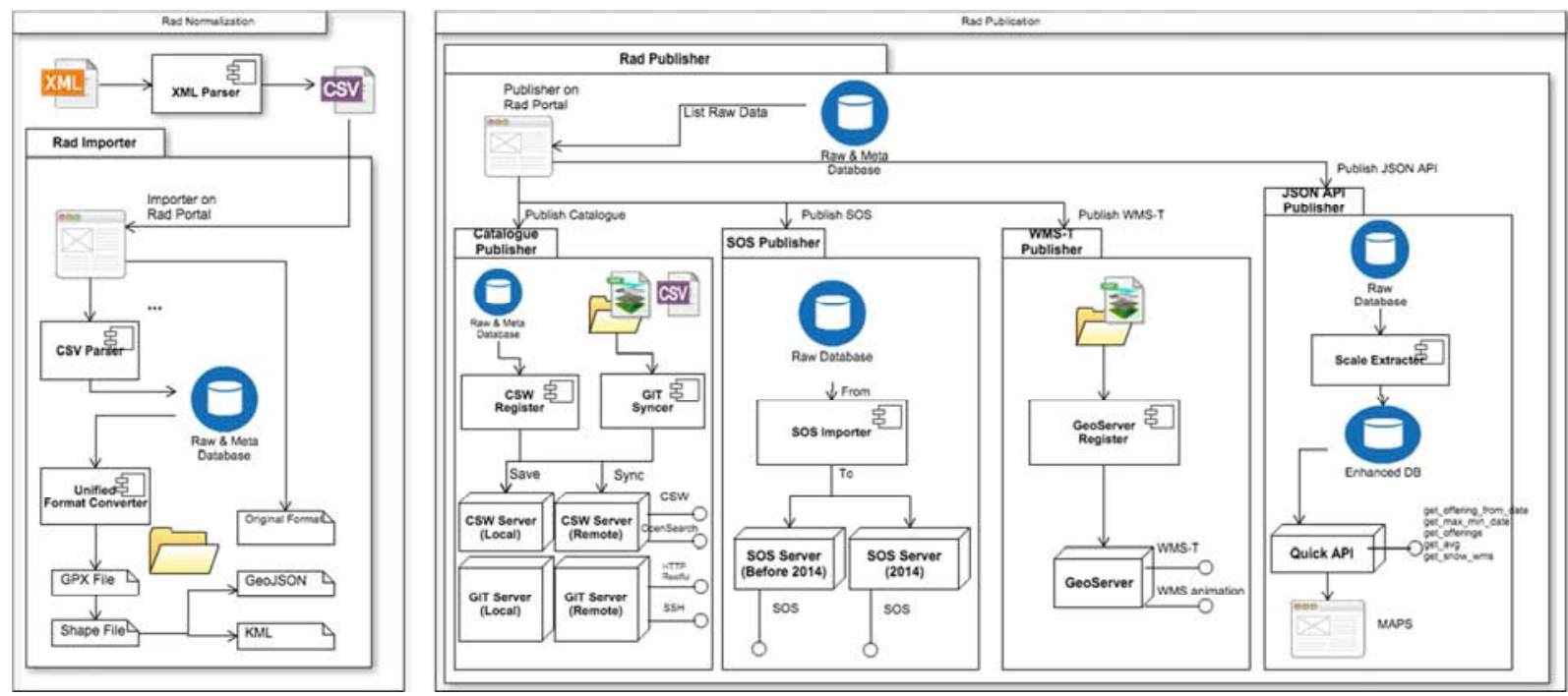

Figure 2. Overview of two major phases of prototype framework.

\section{Results and Discussion}

\subsection{Radiation Normalization Phase}

Radiation normalization is a phase concentrating on the process of importing radiation monitoring data as a normalized data format either into a database or as other file formats like GPX, SHP. Normally, the original monitoring information is stored in CSV or XML format causing some difficulties for data normalization process. Therefore, this 
project developed a simple interface for this task accepting both file types as input (Figure 3). During the normalization task, several important values were extracted from the source files, for instance date, coordinates or surveillance results and then stored in a database called normalized database or raw database. Finally, the system converted these imported records into GPX files for exchange purpose in the future. At the meantime of GPX files generated, the system also created SHP and JSON files.

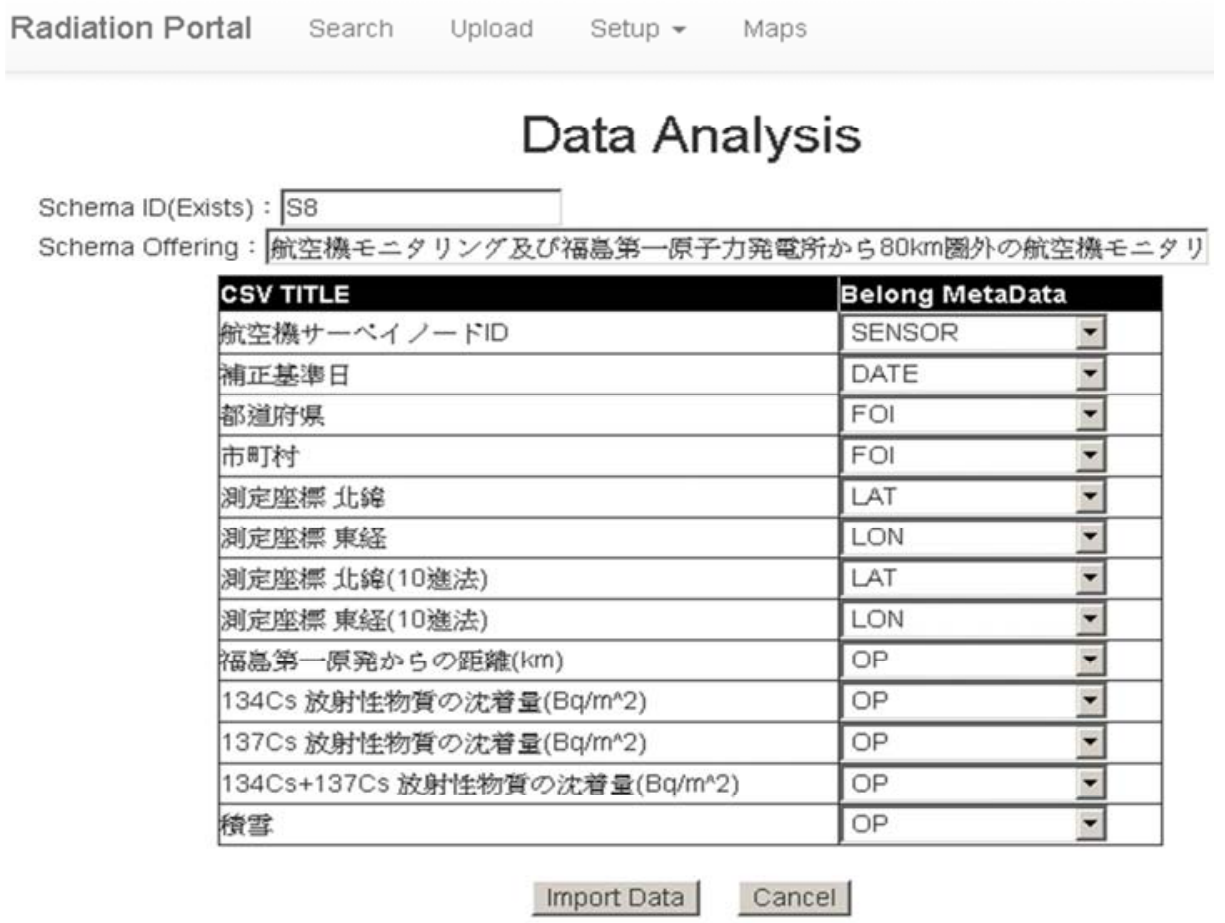

Figure 3. Simple interface for data normalization.

For the process of file uploading and parsing, in case of uploaded files are CSV format, the records in the files will be normalized and stored in the database. The detail processes of CSV uploading and mapping structure with SOS properties are demonstrated by Figure 4.

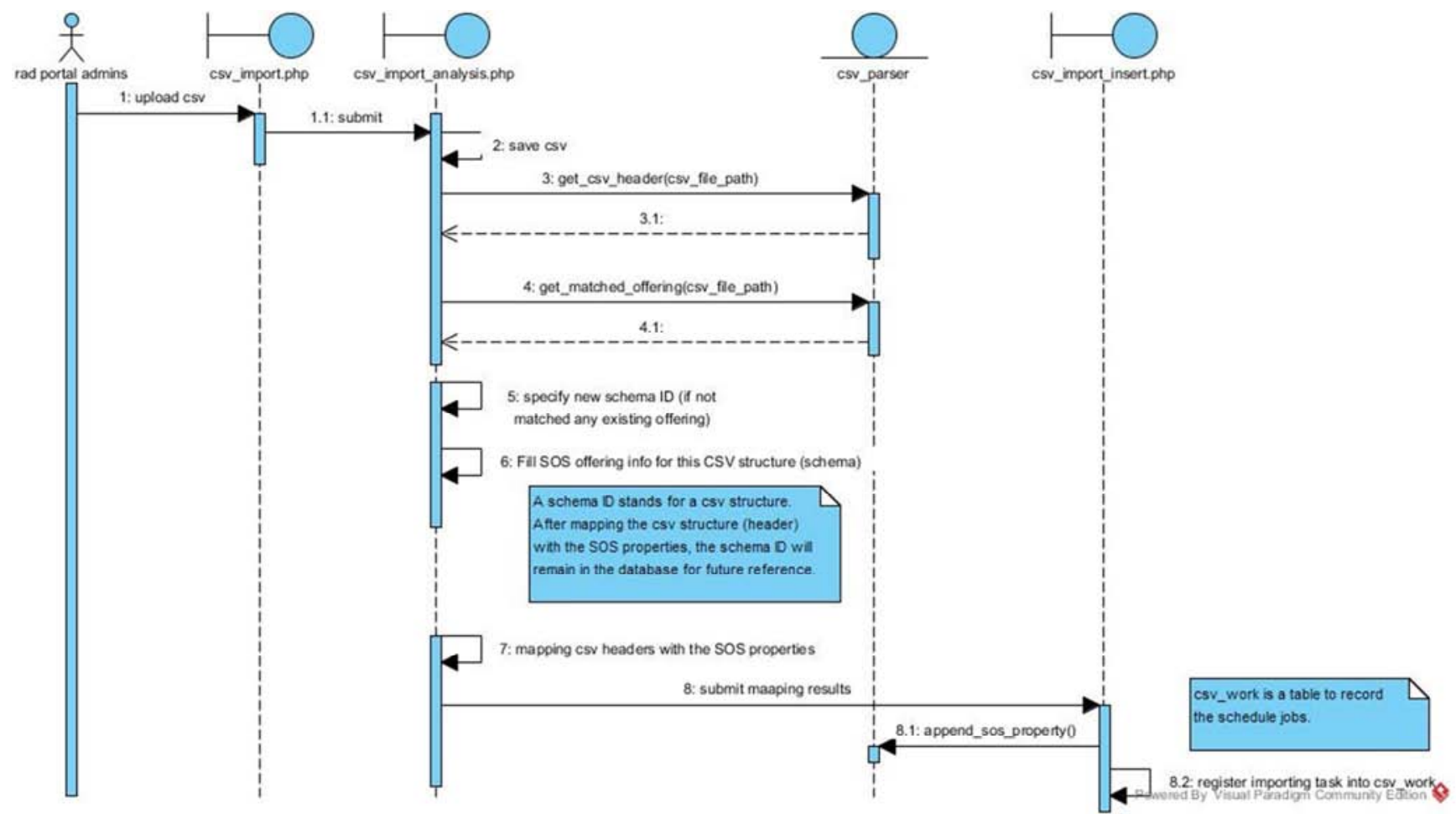




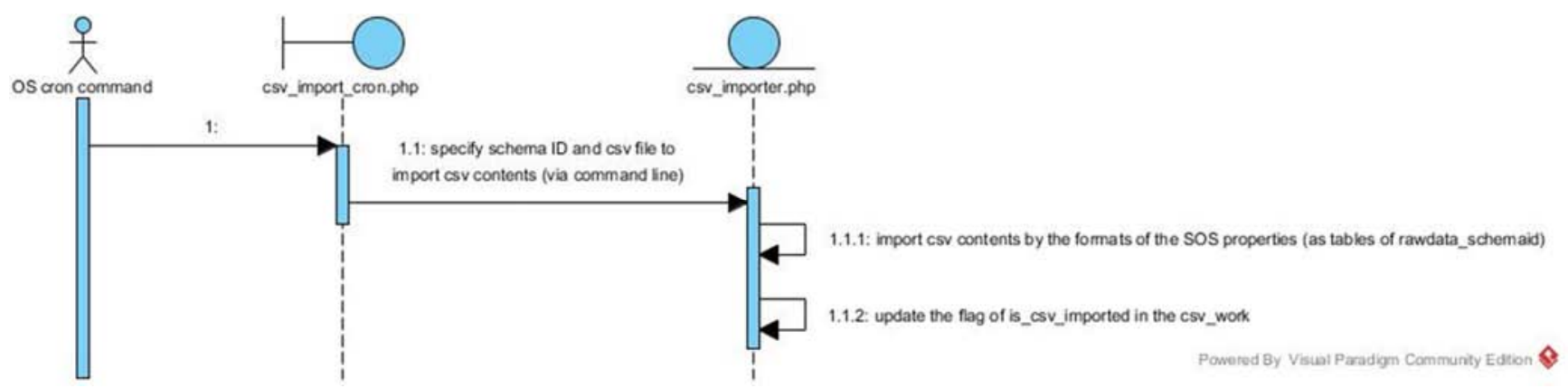

Figure 4. Sequence diagram of CSV uploading and mapping structure with SOS properties.

For XML format, the recommendation is to convert into CSV format and the portal provides two options for handling this task. The first choice is to utilize an XML template file, which must be determined two major "shims" with the first shim for indicating CSV column names and the other for determining which element in the XML is the iteration item, to correctly parse the XML. Figure 5 shows a simple demo for the XML template file with the shims were marked as red color.

In the circumstances of difficulty defining the XML templates, the portal also offers a program to convert the XML files. This program is leveraged to identify elements by counting the number of appearances in which the more number counted, the more possibility it represents the column of the CSV. After this identification, the data will be split into columns and rows.

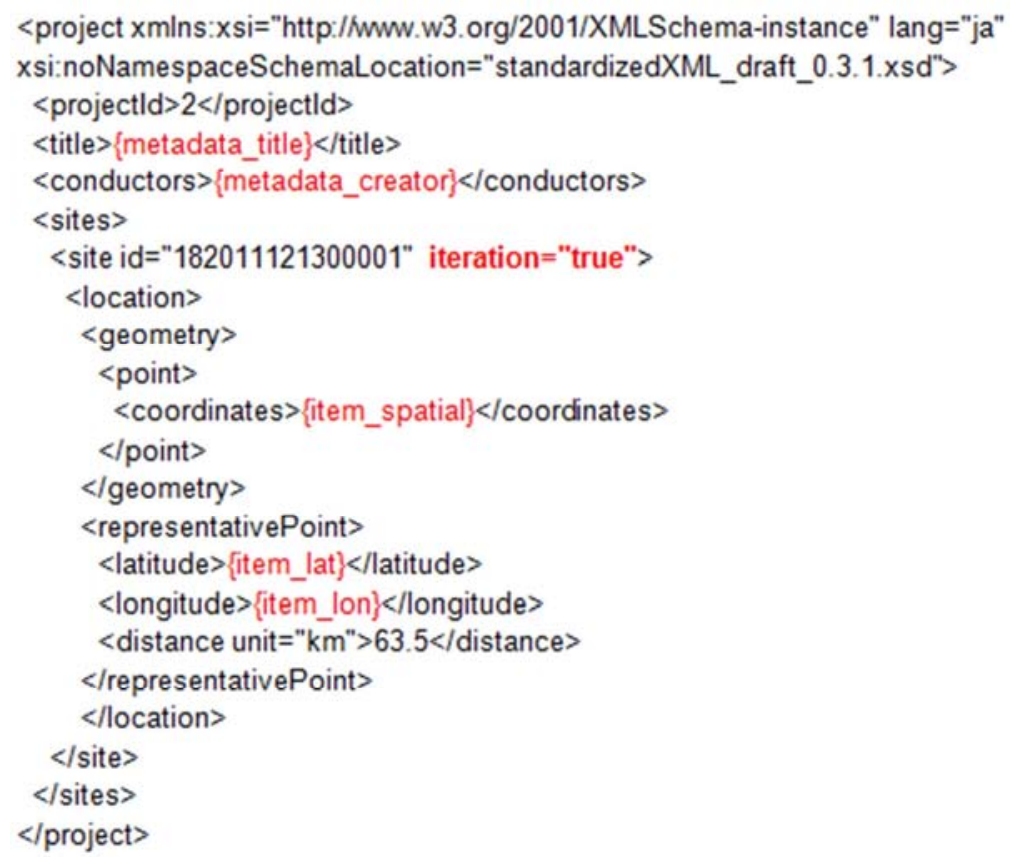

Figure 5. XML template file.

\subsection{Radiation Publication Phase}

\subsubsection{Publish as Catalogue Service}

$\mathrm{CSW}$ is a catalogue service for exploring, querying, and exchanging the metadata from various resources. This study adapted Dublin Core as the standard protocol to describe metadata. The portal was designed to synchronize either metadata or uploaded files to multiple remote servers to deploy same contents to the thirds parties (Figure 6). The metadata, which contain information of location of the files, will be stored in CSW servers while the files will be saved by GIT mechanism and synchronized to remote servers by GIT commands. Each metadata is a collection of records that represent the meaning of multiple radiation files and saved in the database. Therefore, CSW servers were used as the Catalogue service for publishing and exchange these metadata. In order to make the synchronization work, users must setup some required information via setup page of the portal. The detail synchronization flow is shown in Figure 7. 
Radiation Portal Search Upload Setup - Maps

\section{Data Analysis}

Metadata
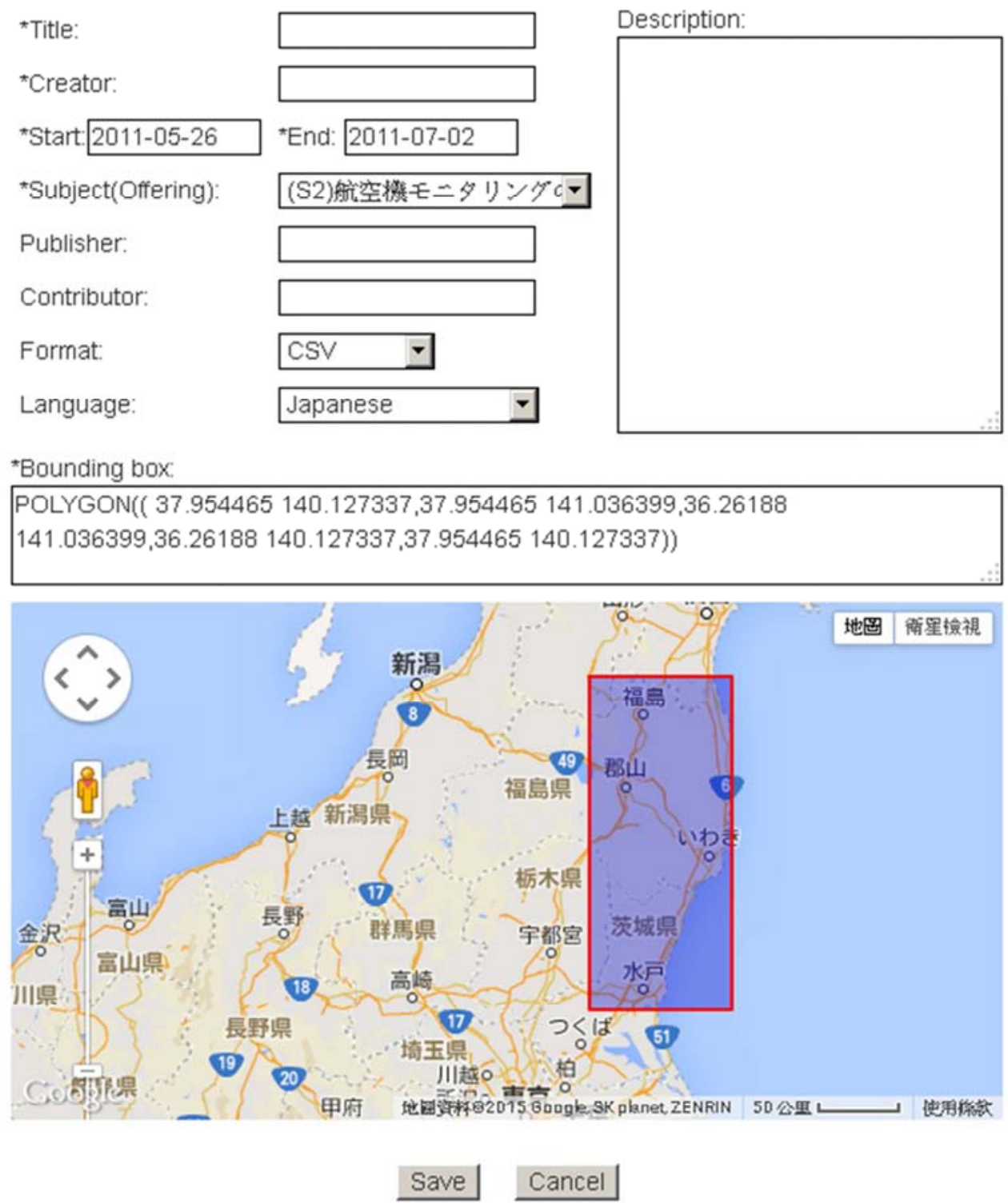

Figure 6. Metadata submission form. 


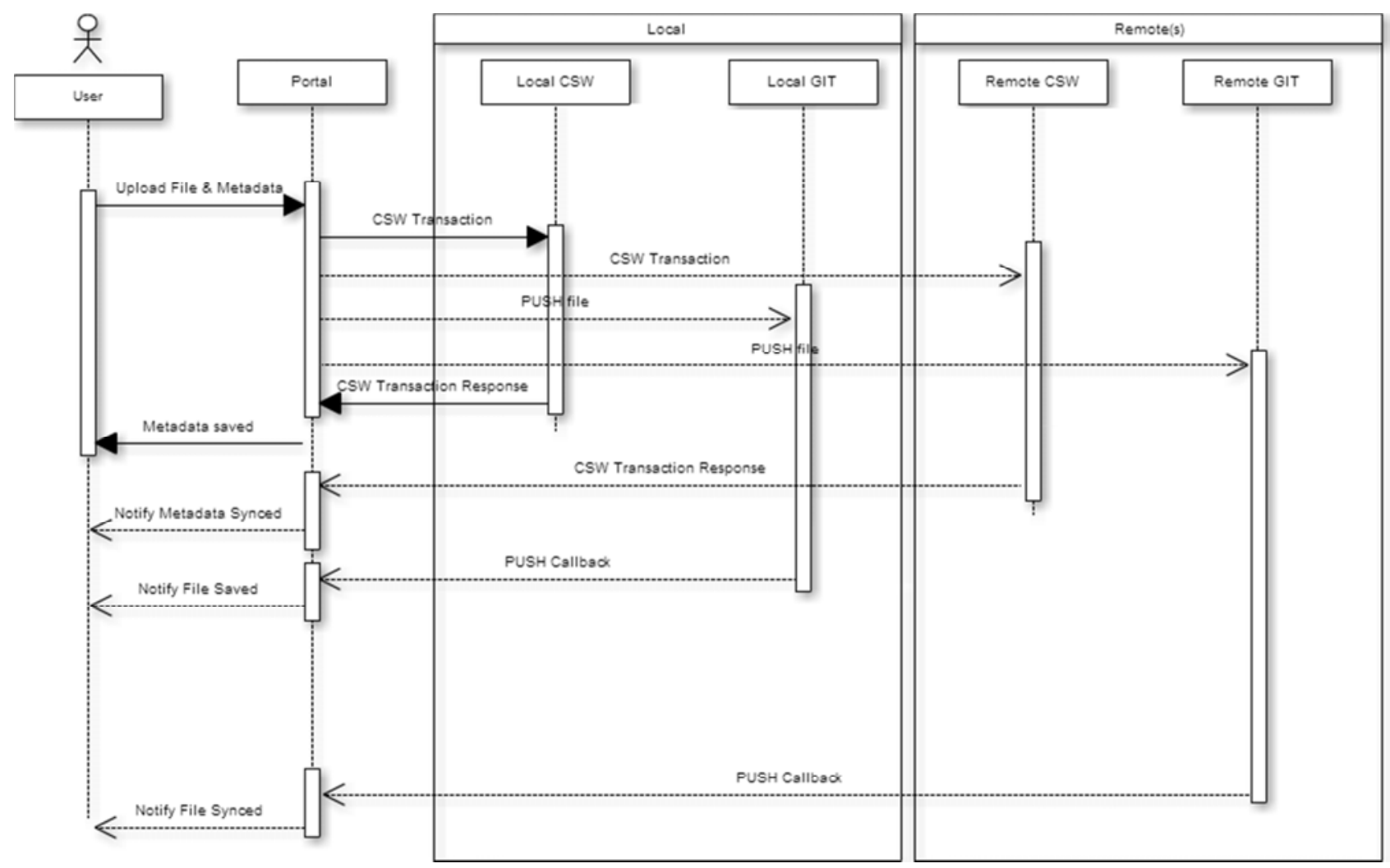

Figure 7. Detail of synchronization process.

\subsubsection{Publish as SOS Service}

SOS is adapted for publishing the observation based on a manageable manner. The portal can publish the raw database as several SOS services. However, the process of insertion might consume a lot of time and thus was divided into two tasks including generation of inserting XML files and post of the XML files to the SOS interface. Figure 8 shows detail SOS service publishing and its interface.

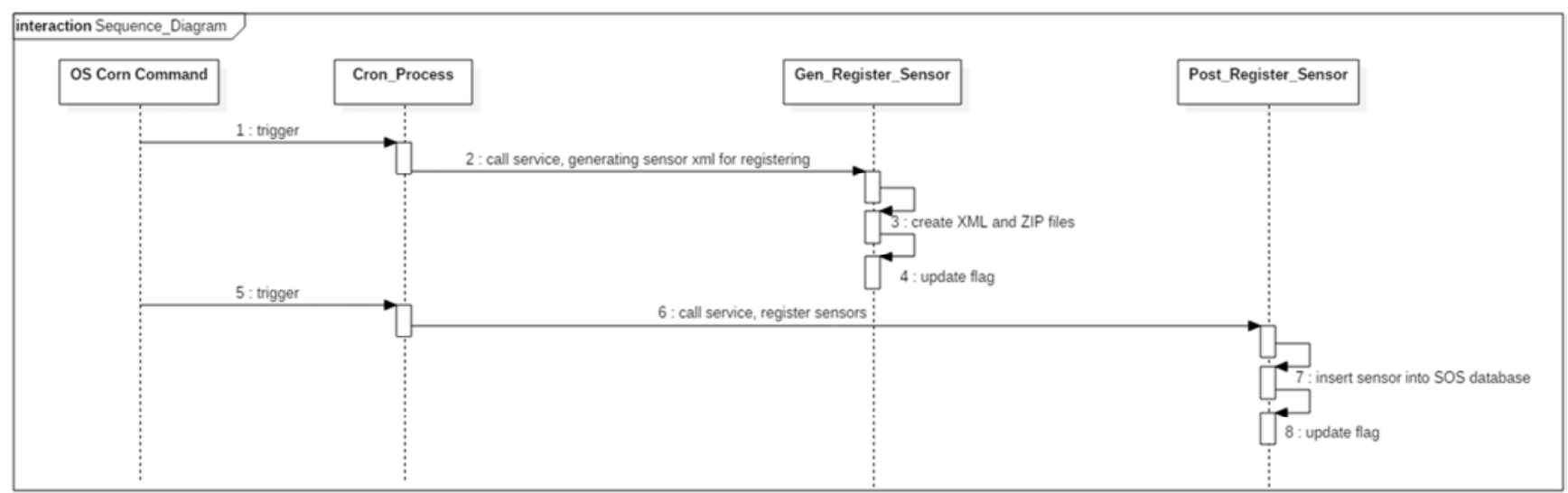

Radiation Portal Search Upload Setup - Maps

Metadata List

\section{Metadata}

ID

D
SOS Insertion Options

Target Sos Servers

SOS POST URL(SOS POSt UrI) •

10 Air Dose Rate Results of the Airborne Monitoring

Survey in the Areas to which Evacuation Orders

$\$ 47$

2013-03-

Have Been Issued

$11 / 2013$

03-11

Figure 8. Detail process of publishing as SOS service and its interface. 


\subsubsection{Publish as JSON API}

For obtaining highest system performance, a JSON API, which provides a quick way to integrate the radiation data with the web apps or other third parties programs supporting JSON format, has been implemented. In order to guarantee the performance of the JSON API, the data is divided by offering names from raw database to different tables in so-called "Enhanced Database". With the purpose of making font-end user interface become more efficient, the portal will reduce unnecessary points under different map scale. Each record in the table will be recalculated the precision of coordinates based on the Japan Mesh Codes, which divided the whole Japan into a geographic grid system with a unique code in different scales. In order to make the statistics more efficient, this system makes a mapping calculation between the mesh level and the map zoom level (Table 1).

\subsubsection{Publish as WMS-T Services}

In this platform, GeoServer - an open source server created for geospatial data sharing using open standards, was utilized to implement the WMS-T standard. In the phase of normalization, the system generated extra files for future exchange including SHP format. The WMS-T publisher will read the metadata from the raw database, register the SHP files into GeoServer and then publish them as WMS service.

Table 1. Japan Mesh Code.

\begin{tabular}{lllllll}
\hline Mesh Abbr & Name & LAT Degree & LON Degree & Grid Length & Scale & Map Zoom Level \\
\hline 1 & Primary mesh & $40^{\prime}$ & 1 & $80 \mathrm{KM}$ & 200,000 & $1-5$ \\
2 & Secondary mesh & $5^{\prime}$ & $70^{\prime \prime}$ & $10 \mathrm{KM}$ & 25,000 & $6-8$ \\
3 & Tertiary mesh & $30^{\prime \prime}$ & $45^{\prime \prime}$ & $1 \mathrm{KM}$ & 2,500 & $9-10$ \\
half & $1 / 2$ mesh & $15^{\prime \prime}$ & $22.5^{\prime \prime}$ & $500 \mathrm{~m}$ & 1,250 & \\
quarter & $1 / 4$ mesh & $7.5^{\prime \prime}$ & $11.25^{\prime \prime}$ & $250 \mathrm{~m}$ & 625 & \\
eighth & $1 / 8$ mesh & $3.75^{\prime \prime}$ & $5.625^{\prime \prime}$ & $125 \mathrm{~m}$ & 313 & \\
tenth & $1 / 10$ mesh & $3^{\prime \prime}$ & $4.5^{\prime \prime}$ & $100 \mathrm{~m}$ & 250 & $11-23$ \\
\hline
\end{tabular}

Bus-as-vehicle monitoring data was adopted as an example for demonstration of time series GIF motion effect (Figure 9).

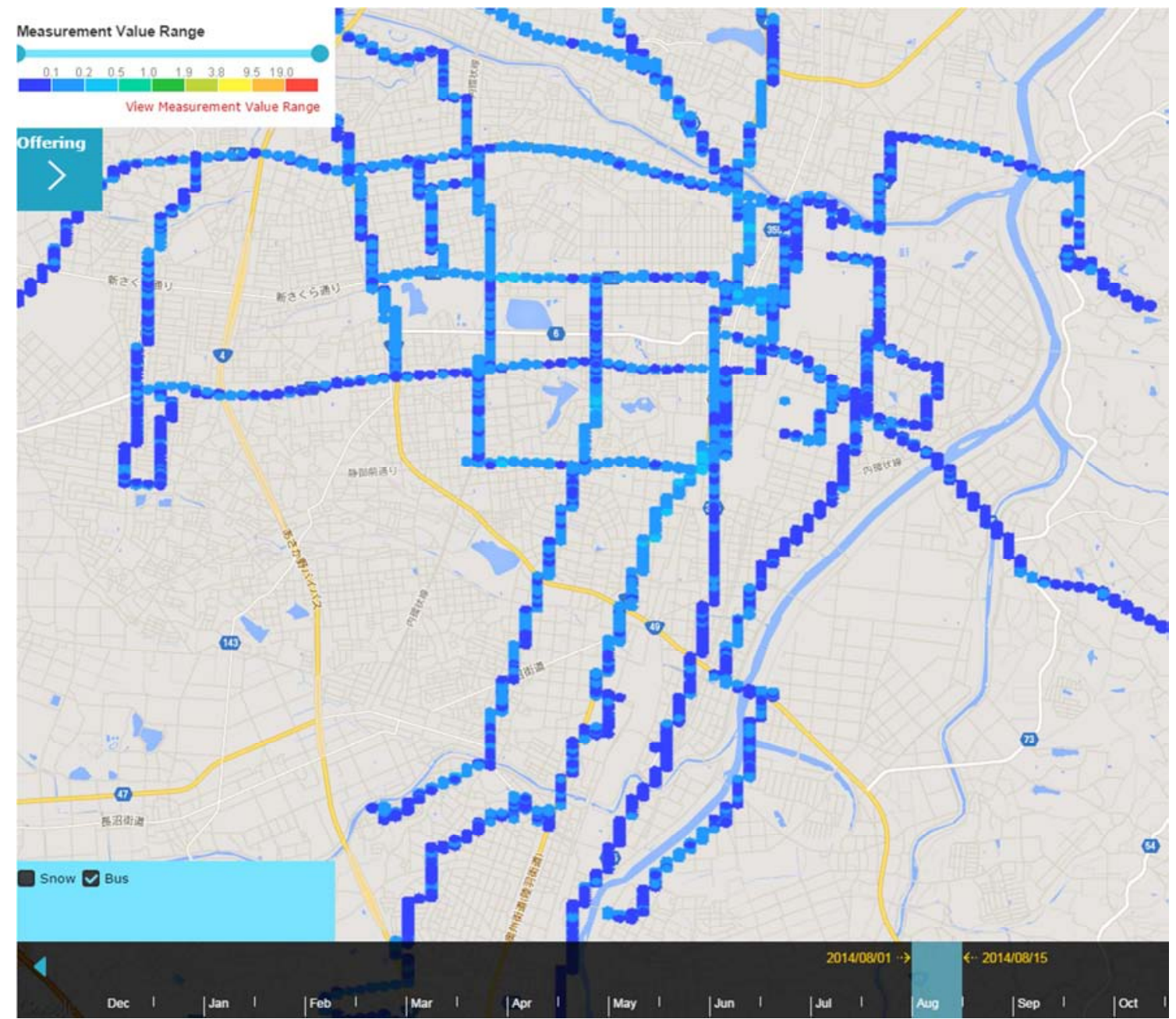

Figure 9. WMS for implementing the motion effect based on time series monitoring data gathered by buses. 


\subsubsection{Visualization Interface for Client}

After publishing the original data as a service, the platform also provides a client interface in which the time series are used as the main axis, and the time segments of the observation are marked on the time axis (Figure 10). Users can view the period of each monitoring task from the timeline as well as query the observation

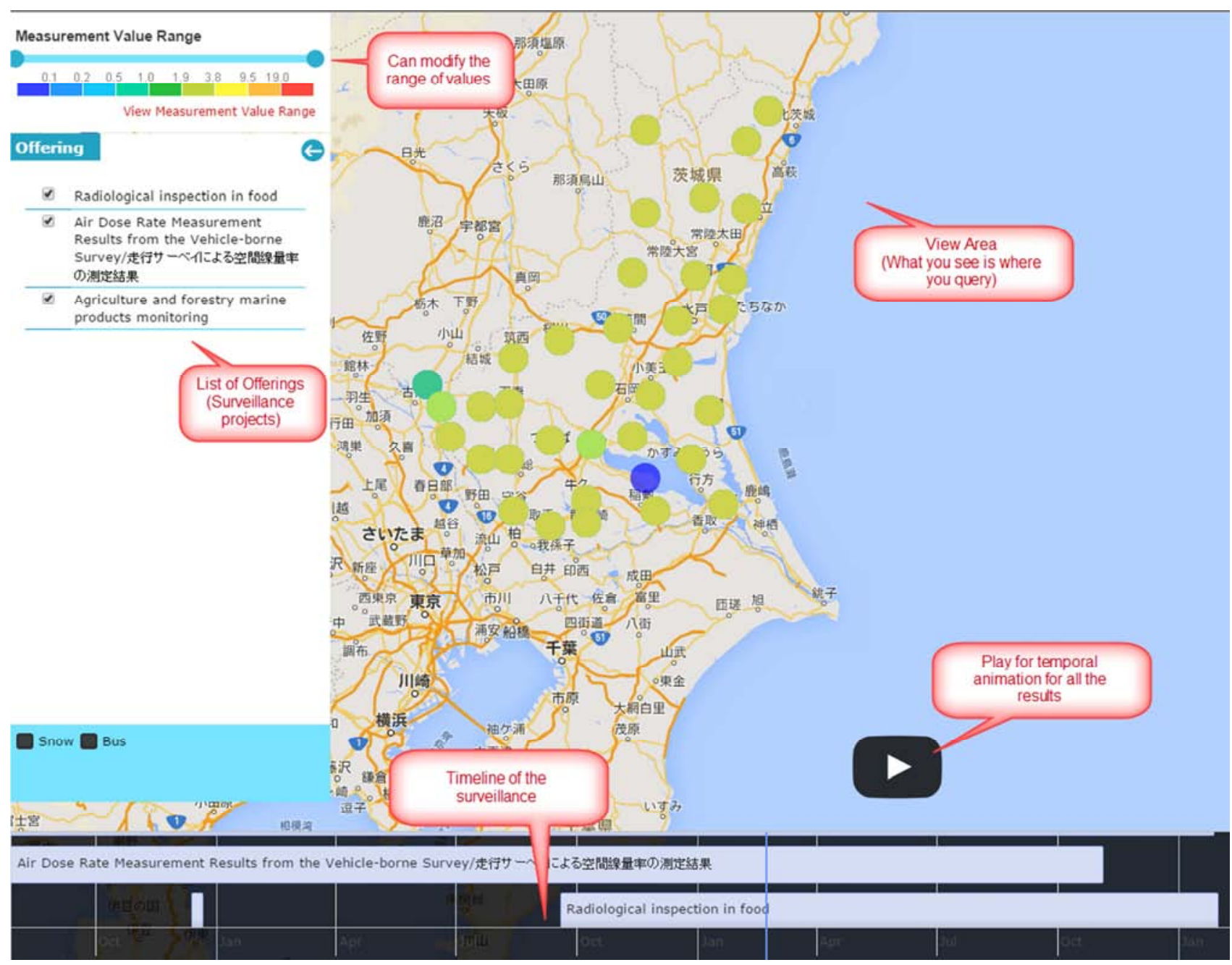

Figure 10. Visualization interface for client.

\section{Conclusions}

The incident at Fukushima Daiichi Nuclear Power Station is one of the most dangerous incidents in the world in terms of nuclear power that affected a larger number of people living near the accident location due to the radioactive materials emission. It has been more than 7 years from the accident with many efforts have been conducted by the government of Japan to determine the level of radioactive contamination and how it has been affected the life of citizens. Utilizing sensor equipment as a means for monitoring and gathering observation data is the selection of many researchers. As a result, the large amount of data from various sensor types has been produced and managed by Japan government. This study has been successfully developed a platform applying some OGC standards to implement the integration of various kinds of sensor monitoring data. A large amount of monitoring data has been processed and exposed to citizens via time series interface that allows users to visualize and query all results. Currently, there are many of the approaches for implementing data interoperability and the result of this study is a contribution to the efforts of applying OGC international standard for dealing with the data sharing issues.

\section{Acknowledgements}

This research was supported by grants from the National Institute of Advanced Industrial Science and Technology, Japan.

\section{References}

[1] Nuclear Emergency Response Headquarters, Government of Japan (2011). Report of Japanese Government to the IAEA Ministerial Conference on Nuclear Safety, The Accident at TEPCO's Fukushima Nuclear Power Stations. http://japan.kantei.go.jp/kan/topics/201106/iaea_houkokusho_ e.html. Cited 13 March 2018. 
[2] Suzuki, S. (2016). Childhood and Adolescent Thyroid Cancer in Fukushima after the Fukushima Daiichi Nuclear Power Plant Accident: 5 Years On. Clinical Oncology, 28(4), 263-271. http://doi.org/https://doi.org/10.1016/j.clon.2015.12.027

[3] Nihei, N. (2016). Monitoring Inspection for Radioactive Substances in Agricultural, Livestock, Forest and Fishery Products in Fukushima Prefecture. In T. M. Nakanishi \& K. Tanoi (Eds.), Agricultural Implications of the Fukushima Nuclear Accident: The First Three Years (pp. 11-21). Tokyo: Springer Japan.

[4] Monma, T., Lurhathaiopath, P., Kawano, Y., Byambasuren, D., Ono, Y., \& Evine, Q. (2015). Developing and Trialing a System to Monitor Radionuclides in Individual Plots of Farmland to Help Reconstruction Farming in Contaminated Areas. In T. Monma, I. Goto, T. Hayashi, H. Tachiya, \& K. Ohsawa (Eds.), Agricultural and Forestry Reconstruction After the Great East Japan Earthquake: Tsunami, Radioactive, and Reputational Damages (pp. 149-167). Tokyo: Springer Japan.

[5] Steinhauser, G. (2017). Monitoring and radioecological characteristics of radiocesium in Japanese beef after the Fukushima nuclear accident. Journal of Radioanalytical and Nuclear Chemistry, 311(2), 1367-1373.

[6] Ohte, N., Murakami, M., Endo, I., Ohashi, M., Iseda, K., Suzuki, T., ... Ishii, N. (2016). Ecosystem Monitoring of Radiocesium Redistribution Dynamics in a Forested Catchment in Fukushima After the Nuclear Power Plant Accident in March 2011. In T. M. Nakanishi \& K. Tanoi (Eds.), Agricultural Implications of the Fukushima Nuclear Accident: The First Three Years (pp. 175-188). Tokyo: Springer Japan.

[7] Matsuda, N., Mikami, S., Sato, T., \& Saito, K. (2017). Measurements of air dose rates in and around houses in the Fukushima Prefecture in Japan after the Fukushima accident. Journal of Environmental Radioactivity, 166, 427-435. https://doi.org/https://doi.org/10.1016/j.jenvrad.2016.03.012.

[8] Martin, P. G., Kwong, S., Smith, N. T., Yamashiki, Y., Payton, O. D., Russell-Pavier, F. S., ... Scott, T. B. (2016). 3D unmanned aerial vehicle radiation mapping for assessing contaminant distribution and mobility. International Journal of Applied Earth Observation and Geoinformation, 52, 12-19. https://doi.org/https://doi.org/10.1016/j.jag.2016.05.007.

[9] Hussain, A., Wu, W., Anzaldi, G., \& Abecker, A. (2015). Implementation of OGC Compliant Framework for Data Integration in Water Distribution System. Procedia Engineering, 119, 1366-1374. https://doi.org/https://doi.org/10.1016/j.proeng.2015.08.984.

[10] Anzaldi, Gabriel; Wu, Wenyan; Abecker, Andreas; Rubión, Edgar; Corchero, Aitor; Hussain, Ambreen; and Quenzer,
Michael,. (2014). Integration Of Water Supply Distribution Systems By Using Interoperable Standards To Make Effective Decisions. CUNY Academic Works. https://academicworks.cuny.edu/cc_conf_hic/421.

[11] Song, X., Rui, X., Hou, W., \& Tan, H. (2008). An OGC standard-oriented architecture for distributed coal mine map services. Journal of China University of Mining and Technology, 18(3), 381-385. https://doi.org/https://doi.org/10.1016/S1006-1266(08)600802

[12] Percivall, G. (2010). The application of open standards to enhance the interoperability of geoscience information. International Journal of Digital Earth, 3(sup1), 14-30. https://doi.org/10.1080/17538941003792751.

[13] Pons, X., \& Masó, J. (2016). A comprehensive open package format for preservation and distribution of geospatial data and metadata. Computers \& Geosciences, 97, 89-97. https://doi.org/https://doi.org/10.1016/j.cageo.2016.09.001.

[14] Evangelidis, K., Ntouros, K., Makridis, S., \& Papatheodorou, C. (2014). Geospatial services in the Cloud. Computers \& Geosciences, 63, 116122.https://doi.org/https://doi.org/10.1016/j.cageo.2013.10.00 7.

[15] Simons, B. A., Yu, J., \& Leighton, B. (2016). WESCML: A Data Standard for Exchanging Water and Energy Supply and Consumption Data. Procedia Engineering, 154, 215-222. https://doi.org/https://doi.org/10.1016/j.proeng.2016.07.451.

[16] Blower, J. D., Gemmell, A. L., Griffiths, G. H., Haines, K., Santokhee, A., \& Yang, X. (2013). A Web Map Service implementation for the visualization of multidimensional gridded environmental data. Environmental Modelling \& Software, $\quad 47$, 218-224. https://doi.org/https://doi.org/10.1016/j.envsoft.2013.04.002.

[17] Wagemann, J., Clements, O., Figuera, R. M., Rossi, A. P., \& Mantovani, S. (2018). Geospatial web services pave new ways for server-based on-demand access and processing of Big Earth Data. International Journal of Digital Earth, 11(1), 7-25. https://doi.org/10.1080/17538947.2017.1351583.

[18] Han, W., Di, L., Yu, G., Shao, Y., \& Kang, L. (2016). Investigating metrics of geospatial web services: The case of a CEOS federated catalog service for earth observation data. Computers \& Geosciences, 92, 1-8. https://doi.org/https://doi.org/10.1016/j.cageo.2016.04.005.

[19] Fukushima prefectureal government (2018). Fukushima Prefecture Outlines. Available via http://www.pref.fukushima.lg.jp/site/portal-english/en05-01.ht ml. Accessed 3 May 2018. 